Nonlinear Processes in Geophysics (2005) 12: 257-267

SRef-ID: $1607-7946 / \mathrm{npg} / 2005-12-257$

European Geosciences Union

(c) 2005 Author(s). This work is licensed

under a Creative Commons License.

\title{
Data assimilation for plume models
}

\author{
C. A. Hier Majumder ${ }^{1,4}$, E. Bélanger ${ }^{2}$, S. DeRosier ${ }^{3,4, ~}{ }^{*}$, D. A. Yuen ${ }^{3,4}$, and A. P. Vincent ${ }^{2}$ \\ ${ }^{1}$ Computational Physics Group, Earth Science Division, Lawrence Livermore National Laboratory, Livermore, CA, USA \\ ${ }^{2}$ Département de Physique, Université de Montréal, Montréal, Québec, Canada \\ ${ }^{3}$ Department of Geology and Geophysics, University of Minnesota, Minneapolis, Minnesota, USA \\ ${ }^{4}$ Minnesota Supercomputing Institute, University of Minnesota, Minneapolis, Minnesota, USA \\ *now at: Department of Earth and Space Sciences, University of Washington, Seattle, Washington, USA
}

Received: 9 August 2004 - Revised: 7 December 2004 - Accepted: 8 December 2004 - Published: 9 February 2005

\begin{abstract}
We use a four-dimensional variational data assimilation (4D-VAR) algorithm to observe the growth of 2-D plumes from a point heat source. In order to test the predictability of the 4D-VAR technique for 2-D plumes, we perturb the initial conditions and compare the resulting predictions to the predictions given by a direct numerical simulation (DNS) without any 4D-VAR correction. We have studied plumes in fluids with Rayleigh numbers between $10^{6}$ and $10^{7}$ and Prandtl numbers between 0.7 and 70, and we find the quality of the prediction to have a definite dependence on both the Rayleigh and Prandtl numbers. As the Rayleigh number is increased, so is the quality of the prediction, due to an increase of the inertial effects in the adjoint equations for momentum and energy. The horizon predictability time, or how far into the future the 4D-VAR method can predict, decreases as Rayleigh number increases. The quality of the prediction is decreased as Prandtl number increases, however. Quality also decreases with increased prediction time.
\end{abstract}

\section{Introduction}

Scientists often do not know the exact initial conditions for a numerical simulation. For example, a meteorologist can never know the exact state of the atmosphere at a given time. Therefore, there will always be errors in the initial conditions for meteorological forecasts (Daley, 1991). Due to the instability of the atmosphere with respect to small amplitude perturbations, two slightly different states may evolve into appreciably different states (Lorenz, 1982). This means that atmospheric forecasts have an intrinsic upper bound to predictability of about two weeks (Lorenz, 1984). Similarly, geophysicists cannot know the exact position of the continents in the past, nor the full temperature and velocity fields at a given instant. Consequently, any numerical simulation of mantle convection will always have uncertainties in the initial conditions (Bunge et al., 2003; Ismail-Zadeh et al., 2004). It

Correspondence to: C. A. Hier Majumder

(cathy@msi.umn.edu) is even difficult to set initial conditions for simulations modeling the behavior of individual plumes in the present mantle since tomographic data can be noisy, and the resolution can vary greatly with location.

The effect of inaccurate initial conditions can be decreased using variational data assimilation (Courtier et al., 1993). This method was developed by meteorologists to increase the accuracy of weather predictions (Daley, 1991). Using the adjoint equations allows the simulation to move backward in time to correct inaccuracies in the initial conditions (Courtier, 1997; Errico, 1997). For example, a weather forecast is started with a set of initial conditions. These initial conditions consist of measurements of the state of the atmosphere and oceans at a given time. As the simulation is computed, the meteorologists continue to collect more weather data at future times. Variational data assimilation allows them to add these observations into the model. The simulation can then move backward in time to correct the old initial conditions and give results closer to the new observations when the model reaches the point in time at which the new observations were taken. This method allows meteorologists to continually improve the data integrated into the simulation with time and leads to more accurate forecasts. It also allows data that occurs after the analysis time to be integrated into the simulation (Zhu et al., 2003). For example, in climate research one would want to integrate observations about the present state of the atmosphere into a model that provides information about the past state of the atmosphere.

Lack of knowledge about the initial conditions is a common problem in geophysical modeling. Often geophysicists want to use the current state of a system to predict a system's previous state. A common example is locating the source of a groundwater contaminant. This requires one to backward model the present distribution of the contaminant to its source. The adjoint equations provide the ideal means of dealing with these questions because they express the backward probability of a state, or the likelihood of the present state resulting from a previous state (Neupauer and Wilson, 2001). 
The adjoint equations have also been used to find the thermal state of the Earth's mantle in the mid-Cretaceous (100 mya) (Bunge et al., 2003). This mantle study begins with the present state of the mantle as known from seismic tomography. It then moves backward and forward in time while using variational data assimilation to incorporate data from past plate motions and decrease the residuals. The correction process becomes stationary after 100 iterations, and the final output is a thermal picture of the mantle 100 mya.

Four-dimensional variational data assimilation (4D-VAR) takes advantage of the powers of both the adjoint equations and variational data assimilation. It uses a periodic update of the adjoint sensitivity field to integrate time distributed observations into the simulation (Daescu and Navon, 2003). It has been used in simulations of floods in river and dam systems (Bélanger and Vincent, 2005; Bélanger et al., 2003). These simulations need river height as an initial condition. The river height, however, can be quite variable due to precipitation and water discharge throughout the watershed. It is impossible to know in advance the exact river height this type of system will experience. Therefore, the initial river height used is always erroneous. With variational data assimilation the modeler can develop a simulation that is less dependent on the error in the initial river height.

This study focuses on the behavior of thermal plumes. Plumes of type are important to a wide variety of phenomena, including mantle convection (Kaminski and Jaupart, 2003; Lithgow-Bertelloni et al., 2001), deep sea thermals (Lavelle, 1997), solar convection (Rast, 1998, 2000), and fires (Cetegen et al., 1998). Many advances have been made in the past ten years on the development of laboratory experimental techniques for the study of finite Prandtl plumes (Moses et al., 1993; Cetegen et al., 1998; Lithgow-Bertelloni et al., 2001; Kaminski and Jaupart, 2003). The 4D-VAR technique discussed here can be used as an optimal control tool to allow experimentalists to check their ongoing experiments in real time or even as a prediction technique to help determine the expected behavior at some future time. Although the method developed here deals with finite Prandtl plumes, it can be easily adpated for use with the infinite Prandtl equations. This would allow the technique to be used to incorporate seismic tomography data available for mantle plumes (Montelli et al., 2004; Zhao, 2001) into numerical models.

The goal of our study is to use the 4D-VAR method to prove that we can predict the behavior of turbulent, finitePrandtl plumes even if we do not have a complete knowledge of their initial conditions. We know both the initial and final states of a plume with a defined set of initial conditions. We call this data our "observations". We take our defined initial conditions and add a small error to them. We then use the 4D-VAR method to test whether we can predict the behavior of the plume using the erroneous initial conditions. We refer to the plume calculated from the erroneous initial conditions as the "forecast" or "prediction". For a given time, we iterate forward/backward and look at the residuals between the observation and forecast after the iterations are done. We vary the time to test how long we can accurately predict the behavior of a plume whose initial conditions are not exactly known. We vary the Rayleigh number from $1 \times 10^{6}$ to $3 \times 10^{7}$ and the Prandtl number from 0.7 to 70 in order to test how the predictability changes with Rayleigh and Prandtl number.

\section{The 4D-VAR technique and the equations of the plume}

The 4D-VAR variational assimilation method can be described in the following way. First, a cost function is conceived to measure the error between the forecast and the observations (Talagrand and Courtier, 1987). Then the adjoint equations, which are used to evaluate the gradient of this cost function, are obtained by applying a variational procedure to the Lagrangian problem (Courtier et al., 1993). The cost function and its gradient are minimized by a minimization algorithm, such as the steepest descent (Burden and Faires, 1993), in order to find the optimal initial conditions that will give the optimal forecast.

\subsection{The cost function}

When using a variational method, it is necessary to write the problem as a functional that one wants to minimize. This functional is known as the cost function. It is a measure of the error between the observations and predictions that one wants to minimize. In general, the cost function is written:

$J=\int_{t_{1}}^{t_{2}} \int_{\Omega} f(\vec{\Psi}, \boldsymbol{x}, t) d \boldsymbol{x} d t$

where $f(\boldsymbol{\Psi}, \boldsymbol{x}, t)$ is a scalar function defined on a domain, $\Omega$, and a time interval, $\left[t_{1}, t_{2}\right]$ (Sanders and Katopodes, 2000). $f(\boldsymbol{\Psi}, \boldsymbol{x}, t)$ is a function of $\boldsymbol{\Psi}$ that represents the state variables, such as the speed or the temperature. In this study, the cost function used is:

$J=\frac{1}{2} \int_{\Omega} \int_{t_{1}}^{t_{2}}\left(H_{\mathrm{cal}}-H_{\mathrm{obs}}\right)\left(H_{\mathrm{cal}}-H_{\mathrm{obs}}\right) d t d \boldsymbol{x}$,

where $H_{\text {cal }}$ is the forecasted convective heat flux, and $H_{\mathrm{obs}}$ is the observed convective heat flux. The heat flux is calculated at each grid point by taking the vertical velocity, $v_{z}$, and the temperature, $T$ :

$H=v_{z} T$.

Our cost function is similar to the objective functional of the temperature used by Ismail-Zadeh et al. (2004) except that we took into account errors in both the velocity and temperature field by using the heat flow.

\subsection{The adjoint equations}

The 4D-VAR problem can be formulated as follows: we want to find the trajectory in space-time for the variables of state $\boldsymbol{\Psi}$ that minimize the cost function (Eq. 2) while obeying the physical equations $\mathcal{E}(\boldsymbol{\Psi}, \boldsymbol{x}, t)=0$, which act as the constraints. Therefore, this is a problem of minimization with constraints (Talagrand and Courtier, 1987). 
Usually, when trying to solve a problem with constraints, one uses the Lagrangian. The formulation of the undetermined Lagrangian multipliers are constructed for the system that one wants to study:

$\mathcal{L}(\vec{\Psi}, \vec{\lambda})=J(\vec{\Psi})+\int_{t_{1}}^{t_{2}} \int_{\Omega} \vec{\lambda}(\boldsymbol{x}, t) \cdot \mathcal{E}(\vec{\Psi}, \boldsymbol{x}, t) d \boldsymbol{x} d t$

where $J(\Psi)$ is the cost function and $\lambda(\boldsymbol{x}, t)$ are the undetermined Lagrangian multipliers, also called the adjoint variables (Sanders and Katopodes, 1999). It has been demonstrated that finding the stationary point of the cost function with the constraints $\mathcal{E}(\boldsymbol{\Psi}, \boldsymbol{x}, t)=0$ is equivalent to finding the stationary points of the Lagrangian with respect of $\boldsymbol{\Psi}$ and $\lambda$ (Dimet and Talagrand, 1986).

When we minimize the Lagrangian, the stationary points that we want to find are saddle points; rather than the absolute maximums or minimums (Dimet and Talagrand, 1986). In order to accomplish this task, we will apply the variational operator $\delta$ to the Lagrangian. Here, the displacement directions are the physical variables of the system as well as the adjoint variables. Taking the variation of the Lagrangian, we obtain:

$$
\begin{aligned}
\delta \mathcal{L} & =\vec{\nabla}_{\vec{\Psi}} \mathcal{L} \cdot \delta \vec{\Psi}+\vec{\nabla} \vec{\lambda}_{\vec{\lambda}} \mathcal{L} \cdot \delta \vec{\lambda} \\
& =\frac{\partial \mathcal{L}}{\partial \vec{\Psi}} \delta \vec{\Psi}+\frac{\partial \mathcal{L}}{\partial \vec{\lambda}} \delta \vec{\lambda} .
\end{aligned}
$$

Also note that we have linearized the problem in the same step (Ehrendorfer, 1992). For an arbitrary displacement $(\delta \boldsymbol{\Psi}, \delta \boldsymbol{\lambda})$, we are at a minimum only if $\delta \mathcal{L}=0$ (Daley, 1991). This indicates that the derivative of the Lagrangian with respect to each direction must be zero:

$\frac{\partial \mathcal{L}}{\partial \vec{\lambda}}=\mathcal{E}(\vec{\Psi}, \boldsymbol{x}, t)=0$

and

$$
\frac{\partial \mathcal{L}}{\partial \vec{\Psi}}=\operatorname{Adj}(\vec{\lambda})+\frac{\partial J}{\partial \vec{\Psi}}=0
$$

where $\operatorname{Adj}(\lambda)$ represents the adjoint equations after integration by parts (Schröter et al., 1993). Note that Eq. (6) is the system of equations that one had at the beginning and that Eqs. (6) and (7) are the Euler-Lagrange equations (Dimet and Talagrand, 1986).

Unfortunately, an efficient means for directly solving the Euler-Lagrange equations does not exist. This situation forces us to reformulate our problem to one without constraints (Talagrand and Courtier, 1987). Since the physical equations of the model are deterministic, it is evident that the state of the system at the time of the observations depends only on the initial conditions, $\boldsymbol{\Psi}_{0}$, of the system. Therefore, the cost function is an implicit function of the initial conditions. It is by varying the initial conditions that we will solve the physical equations while minimizing the cost function (Ehrendorfer, 1992). According the theory of optimal control (Lions, 1968), the control variables of the problem are

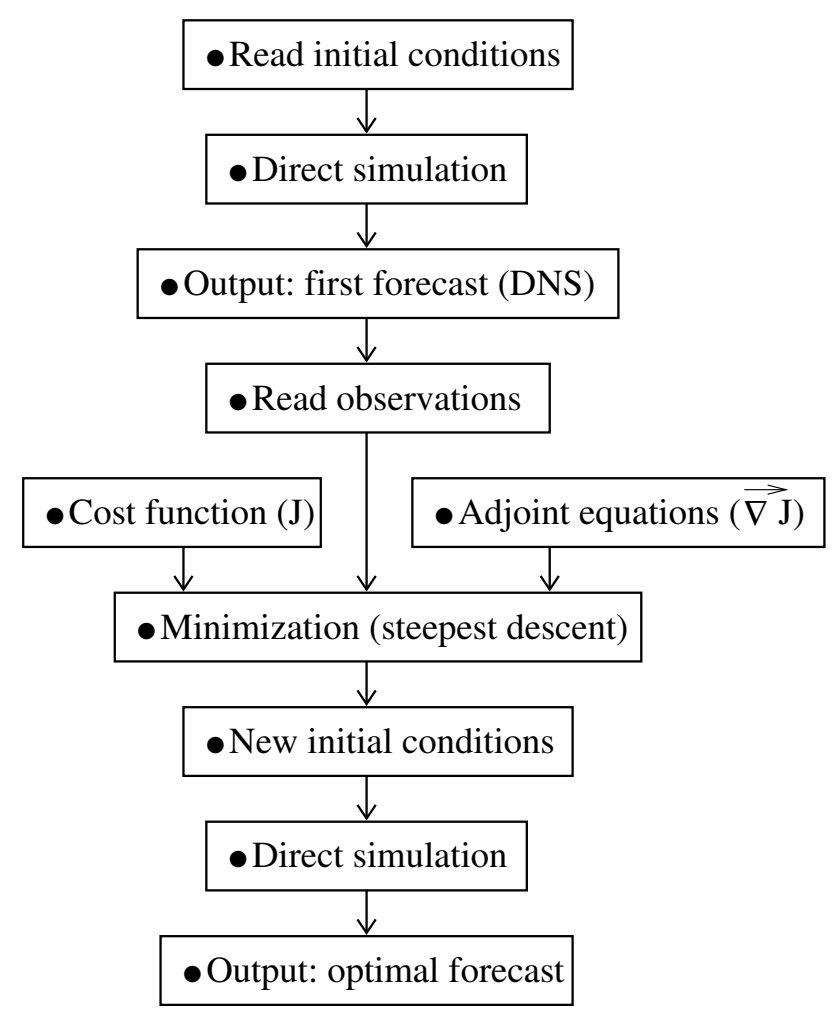

Fig. 1. Algorithm for the 4D-VAR method.

the initial conditions. One can also use the boundary conditions as control variables (Schröter et al., 1993). In our problem, we have removed the constraints since no restrictions are applied to the initial conditions.

Finally, the gradient of the cost function (Eq. 2) with respect to the initial vertical heat flux is given by the adjoint variables evaluated at time $\tau=t_{2}$ (Courtier, 1997):

$\nabla J_{u_{0}}=u^{*}\left(x, z, \tau=t_{2}\right)$
$\nabla J_{w_{0}}=w^{*}\left(x, z, \tau=t_{2}\right)$
$\nabla J_{P_{0}}=P^{*}\left(x, z, \tau=t_{2}\right)$
$\nabla J_{T_{0}}=T^{*}\left(x, z, \tau=t_{2}\right)$.

\subsection{The 4D-VAR data assimilation algorithm}

Starting from initial conditions obtained by current experimental observations or a previous numerical simulation, a direct simulation generates a traditional (DNS) forecast (Fig. 1). After reading the observations taken at the end of the forecast period, the initial error between the forecast and the observations is calculated. This initial error will provide a first guess to a minimization algorithm.

In this theoretical study, we needed to vary the accuracy and the input parameters to test the 4D-VAR technique. Since we were testing the validity of the technique, we did not use real data. Many experimental studies have been conducted on the behavior of finite Prandtl number plumes (Kaminski and Jaupart, 2003; Lithgow-Bertelloni 
et al., 2001; Cetegen et al., 1998; Moses et al., 1993). The 4D-VAR method introduced here will allow integration of experimental studies into numerical models. The technique could also be used to incorporate the tomographic data available of mantle plumes (Montelli et al., 2004; Zhao, 2001).

For the data assimilation run, we have perturbed the initial conditions with a sinusoidal function. A direct simulation with the perturbed initial conditions permits us to obtain a simple forecast. After reading the observations (obtained previously), we calculate the initial error between the forecast and the observations. Then, we use a minimization algorithm, such as the steepest descent, in order to minimize the cost function (Eq. 2) with the aid of its gradient (Sect. 2.2). When the minimum has been found, we have the new initial conditions. These initial conditions are optimal because a second direct simulation using them will be an optimal forecast. The final error between this forecast and the observations will be minimal.

\subsection{The direct equations for a localized thermal plume}

The physical equations describing Rayleigh-Bénard convection with the Boussinesq approximation that we used are:

$\nabla \cdot v=0$

$\frac{\partial \boldsymbol{v}}{\partial t}=\boldsymbol{v} \times \boldsymbol{\omega}-\nabla P+\frac{1}{\operatorname{Re}} \nabla^{2} \boldsymbol{v}+T \hat{e}_{z}$

$\frac{\partial T}{\partial t}=-\boldsymbol{v} \cdot \nabla T+\frac{1}{\mathrm{Pe}} \nabla^{2} T$

where $\omega$ is the vorticity (Hier Majumder et al., 2004). The equations have been nondimensionalized with the free-fall velocity:

$U=\sqrt{\alpha g \Delta T L_{z}}$,

where $\alpha$ is the coefficient of thermal expansion, $g$ is the gravitational acceleration, $\Delta T$ is the initial temperature difference between the heating point and the surrounding fluid, and $L_{z}$ is the box height. The Rayleigh $(R a)$ and Prandtl $(P r)$ numbers are the nondimensional numbers often used in thermal convection equations. We define $R a$ as:

$R a=\frac{g \alpha \Delta T L_{z}^{3}}{\kappa \nu}$,

where $\kappa$ is the thermal diffusivity and $v$ is the kinematic viscosity. The Prandtl number is defined as:

$\operatorname{Pr}=\frac{v}{\kappa}$.

The nondimensional numbers that appear in Eqs. (13) and (14) are the Reynolds number:

$R e=\frac{U L_{z}}{v}$

and the Péclet number:

$P e=\frac{U L_{z}}{\kappa}$
(Tritton, 1988). The $R e$ and $P e$ are related to the Rayleigh number $(R a)$ and the Prandtl number $(P r)$ of the plume by:

$R a=P e R e$

and

$\operatorname{Pr}=\frac{P e}{R e}$.

2.5 The adjoint equations for a localized thermal plume

Following the method in Sect. 2.2, we obtained the adjoint equations (Marchuk, 1995):

$$
\begin{aligned}
\frac{\partial u^{*}}{\partial \tau} & =\boldsymbol{v} \cdot \nabla u^{*}-u \frac{\partial w^{*}}{\partial z}+w \frac{\partial w^{*}}{\partial x}+\frac{1}{\operatorname{Re}} \nabla^{2} u^{*}+u \nabla^{2} P^{*} \\
& -2 u \frac{\partial^{2} P^{*}}{\partial x^{2}}-2 w \frac{\partial^{2} P^{*}}{\partial x \partial z}+T \frac{\partial T^{*}}{\partial x}-\frac{\partial J}{\partial u} \\
\frac{\partial w^{*}}{\partial \tau} & =\boldsymbol{v} \cdot \nabla w^{*}+u \frac{\partial u^{*}}{\partial z}-w \frac{\partial u^{*}}{\partial x}+\frac{1}{\operatorname{Re}} \nabla^{2} w^{*}+w \nabla^{2} P^{*} \\
& -2 u \frac{\partial^{2} P^{*}}{\partial x \partial z}-2 w \frac{\partial^{2} P^{*}}{\partial z^{2}}+T \frac{\partial T^{*}}{\partial z}-\frac{\partial J}{\partial w} \\
\nabla^{2} P^{*} & =\frac{\partial u^{*}}{\partial x}+\frac{\partial w^{*}}{\partial z}-\frac{\partial J}{\partial P} \\
\frac{\partial T^{*}}{\partial \tau} & =\boldsymbol{v} \cdot \nabla T^{*}+\frac{1}{\operatorname{Pe}} \nabla^{2} T^{*}+w^{*}-\frac{\partial P^{*}}{\partial z}-\frac{\partial J}{\partial T}
\end{aligned}
$$

where $\tau=t_{2}-t$ is the inverse time. The initial conditions are:

$$
\begin{array}{ll}
\delta u\left(x, z,\left.t\right|_{t_{1}}\right)=0 & u *\left(x, z,\left.t\right|_{t_{2}}\right)=0 \\
\delta v\left(x, z,\left.t\right|_{t_{1}}\right)=0 & v *\left(x, z,\left.t\right|_{t_{2}}\right)=0 \\
\delta T\left(x, z,\left.t\right|_{t_{1}}\right)=0 & T *\left(x, z,\left.t\right|_{t_{2}}\right)=0
\end{array}
$$

and the boundary conditions are:

$$
\begin{array}{cl}
u *(0, z, t)=0 & u *\left(L_{x}, z, t\right)=0 \\
v *(0, z, t)=0 & v *\left(L_{x}, z, t\right)=0 \\
T *(0, z, t)=0 & T *\left(L_{x}, z, t\right)=0 \\
P *(0, z, t)=0 & P *\left(L_{x}, z, t\right)=0 \\
u *(x, 0, t)=0 & u *\left(x, L_{z}, t\right)=0 \\
v *(x, 0, t)=0 & v *\left(x, L_{z}, t\right)=0 \\
T *(x, 0, t)=0 & T *\left(x, L_{z}, t\right)=0 \\
P *(x, 0, t)=0 & P *\left(x, L_{z}, t\right)=0 \\
\left.\frac{\partial u^{*}}{\partial x}\right|_{x=0}=0 & \left.\frac{\partial u^{*}}{\partial z}\right|_{z=0}=0 \\
\left.\frac{\partial v^{*}}{\partial x}\right|_{x=0}=0 & \left.\frac{\partial v^{*}}{\partial z}\right|_{z=0}=0 \\
\left.\frac{\partial P^{*}}{\partial x}\right|_{x=0}=0 & \left.\frac{\partial P^{*}}{\partial z}\right|_{z=0}=0 \\
\left.\frac{\partial T^{*}}{\partial x}\right|_{x=0}=0 & \left.\frac{\partial T^{*}}{\partial z}\right|_{z=0}=0
\end{array}
$$




$$
\begin{array}{lll}
\left.\frac{\partial u^{*}}{\partial x}\right|_{x=L_{x}}=0 & \left.\frac{\partial u^{*}}{\partial z}\right|_{z=L_{z}}=0 \\
\left.\frac{\partial v^{*}}{\partial x}\right|_{x=L_{x}}=0 & \left.\frac{\partial v^{*}}{\partial z}\right|_{z=L_{z}}=0 \\
\left.\frac{\partial P^{*}}{\partial x}\right|_{x=L_{x}}=0 & \left.\frac{\partial P^{*}}{\partial z}\right|_{z=L_{z}}=0 \\
\left.\frac{\partial T^{*}}{\partial x}\right|_{x=L_{x}}=0 & \left.\frac{\partial T^{*}}{\partial z}\right|_{z=L_{z}}=0 .
\end{array}
$$

\section{Prediction: what can be expected for the 4D-VAR?}

We start with a plume defined by a temperature and velocity field (Fig. 2a). We use this temperature and velocity field as exact initial conditions for a DNS. We save the temperature and velocity field at each iteration of the DNS to create the observations. We then take the plume defined by the exact initial conditions from the DNS and perturb its convective heat flux. This perturbation produces an error in the initial temperature and velocity fields that needs to be corrected by the 4D-VAR technique (Fig. 2b). The same perturbation is used for all Rayleigh and Prandtl numbers. A grid size of $64 \times 192$ is used for all runs. The timestep and number of iterations is set optimal for a given run. The 4D-VAR algorithm is relatively fast to compute. Individual simulations took no longer than a few hours on an IBM Power4 System: pSeries 690 (Regatta).

We did not use a multigrid or continuous deformation grid so we needed to have the same equally spaced grid for each run throughout time and space. It is desirable to use a coarser grid because it requires less computational time. This can be dangerous, however, in systems with strong nonlinearities. In our case, a turbulence model, such as a large-eddy simulation (LES), was not used. This means that the grid size must be large enough to account for features that occur at the turbulent dissipation scale. Numerical diffusion, an artifact due to a coarse grid, may produce an artificially stable solution over the computational grid. Indeed, if the grid is too large, there is not enough resolution. The small-scale process will not be accounted for, and dissipation will occur purely through numerical diffusion (Roache, 1976). Since the turbulent dissipation at small-scales increases with the turbulent Reynolds number, the grid size must increase in each direction as the turbulent Reynolds number increases:

$N \sim R e_{t}$,

where $N$ is the number of grid points and $R e_{t}$ is the turbulent Reynolds number. Since our grid is static in time and space, it must have adequate resolution to model the maximum turbulent Reynolds number that occurs throughout time and space during the simulation.

To test the predictability of the 4D-VAR method, we ran DNS and 4D-VAR simulations starting with the erroneous initial conditions for both simulations. Simulations were run for $\operatorname{Pr}=7$ at $R a=1 \times 10^{6}, R a=2 \times 10^{6}, R a=3 \times 10^{6}$, and
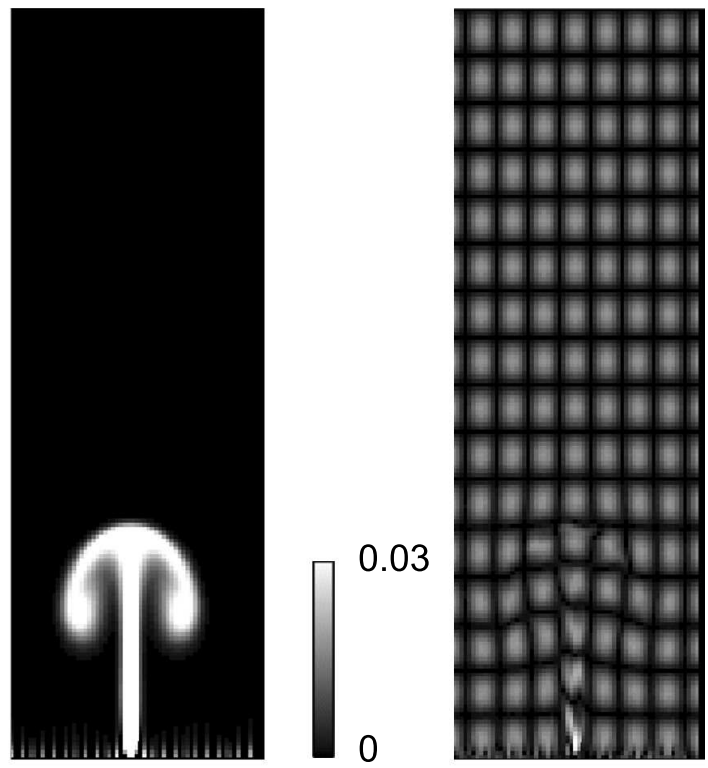

a)

b)

Fig. 2. Plume with $R a=1 \times 10^{6}$ and $\operatorname{Pr}=7$. This shows a plume developed from a 2-D point heat source. We use this plume to define the initial conditions for our simulations. (a) Defined initial temperature field for observations. (b) Error map showing difference between the defined initial temperature and the erroneous initial temperature.

$R a=1 \times 10^{7}$. For $R a=1 \times 10^{6}$, simulations were also run at $\operatorname{Pr}=0.7$ and 70 .

\subsection{Direct numerical simulation}

We used the function $\operatorname{Err}(t)$ to measure the difference between the observations and the predictions created from the perturbed initial conditions. $\operatorname{Err}(t)$ is a measure of the difference between the predicted convective heat flux $\left(H_{c a l}(x, y)\right)$ and the observed convective heat flux $\left(H_{o b s}(x, y)\right)$ after the prediction has been run for a given time, $t$, where time is scaled by the free-fall time:

$t=\frac{t_{d} U}{L_{z}}$

where $t_{d}$ is the dimensional time, $U$ is the free-fall velocity (Eq. 15), and $L_{z}$ is the box height. The error quantity is defined as:

$\operatorname{Err}(t)=<H_{c a l}(x, y)-H_{o b s}(x, y)>(t)$,

where $<>$ is the quadratic mean over $(x, y)$.

For the DNS method, $\operatorname{Err}(t)$ at $t \sim 0$ is the same as the initial error applied to the defined initial conditions to create the erroneous initial conditions. Since no correction has been applied, the prediction is wrong by the same amount as the error added to the initial conditions. As time increases, $\operatorname{Err}(t)$ remains roughly the same. For $\operatorname{Ra}=1 \times 10^{6}$ it begins to increase only after $t=0.3$ (Fig. 3a). The time at which the 


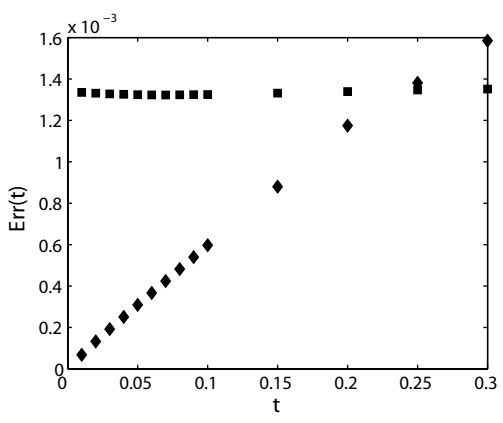

a)

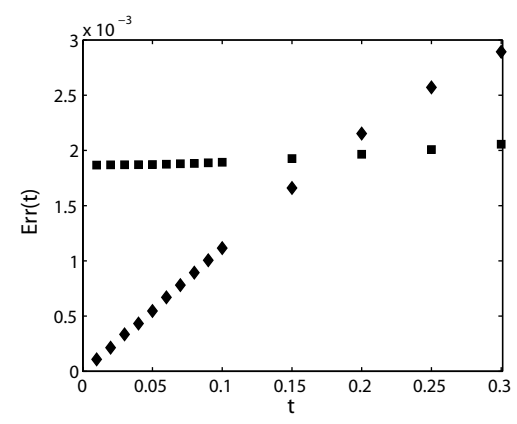

c)

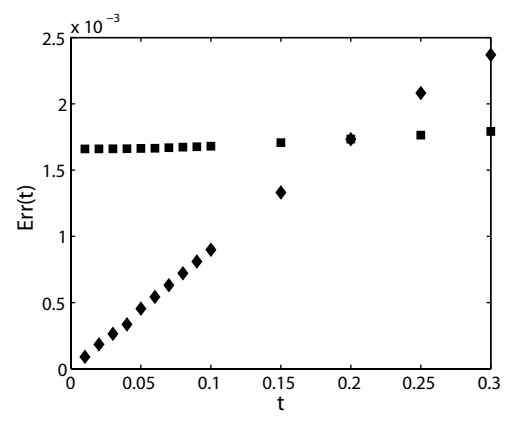

b)

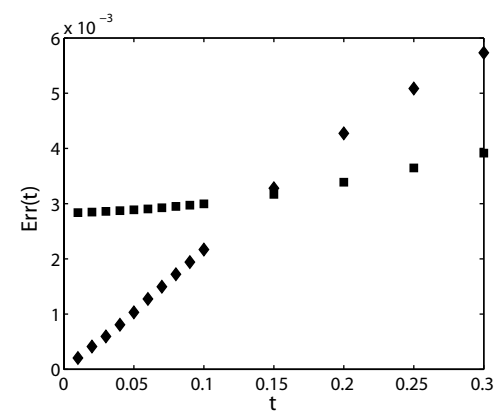

d)

Fig. 3. Comparison between DNS and 4D-VAR at different times after an initial perturbation has been set for $R a=1 \times 10^{6}$. Squares represent $\operatorname{Err}(t)$ when $H_{\text {cal }}$ is calculated using the DNS method. Diamonds represent $\operatorname{Err}(t)$ when $H_{\text {cal }}$ is calculated using the 4D-VAR method. Time, $t$, is scaled by the free-fall time. (a) $R a=1 \times 10^{6}$. (b) $R a=2 \times 10^{6}$. (c) $R a=3 \times 10^{6}$. (d) $R a=1 \times 10^{7}$.

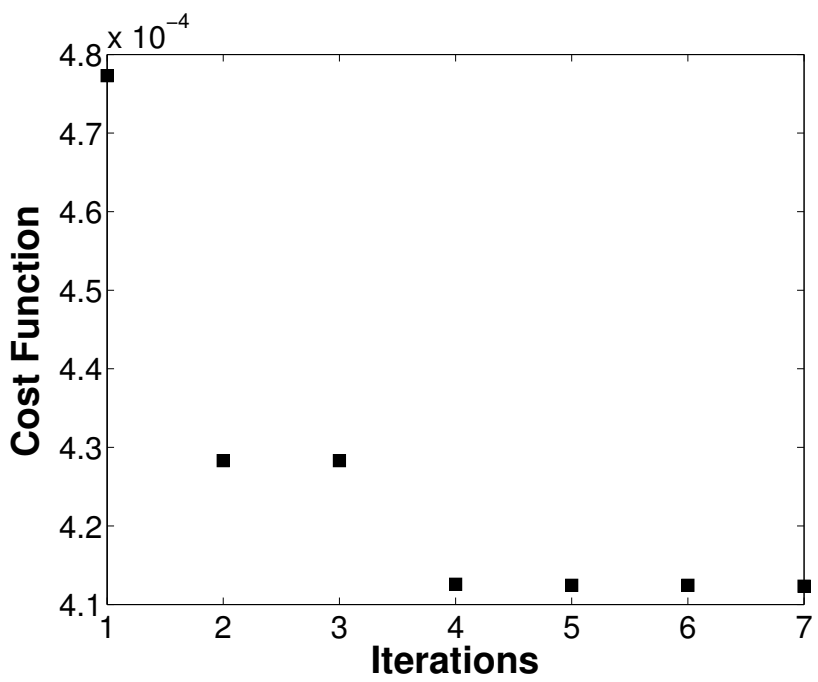

Fig. 4. Minimization of the cost function for $R a=1 \times 10^{6}, t=0.01$.

error begins to increase decreases with $R a$. For $R a=2 \times 10^{6}$, $\operatorname{Err}(t)$ begins to increase after 0.20 (Fig. 3b), and $\operatorname{Err}(t)$ increases after 0.15 for $R a=3 \times 10^{6}$ (Fig. 3c). For $R a=1 \times 10^{7}$, the error starts to increase immediately (Fig. 3d). The error in the direct simulation begins to increase after nonlinear, inertial terms become important. These terms are larger with increasing Rayleigh number. Therefore, we cannot predict as long with increasing Rayleigh number.

\subsection{D-VAR}

The 4D-VAR method is used to decrease the errors due to the perturbed initial conditions. The cost function is minimized with the steepest descent method through use of its gradient calculated from the adjoint equations. We have shown the minimization of the cost function for an example plume of $R a=1 \times 10^{6}, \operatorname{Pr}=7$ (Fig. 4). The free-fall velocity forecast time for this simulation was 0.01 . The cost function is minimized by $14 \%$ over 7 iterations.

The error near $t \sim 0$ in the 4D-VAR case is significantly lower than in the DNS case (Fig. 3). The correction is maximal and the error is minimal a very short time after $t=0$. In fact, $\operatorname{Err}(t)$ is almost 0 . This indicates that the erroneous initial conditions have been fully corrected by the 4D-VAR method. As time is increased, $\operatorname{Err}(t)$ increases, and the quality of the 4D-VAR prediction degrades. At some time, known as the horizon of predictability, the 4D-VAR prediction is no better than the DNS prediction to which no correction was applied.

\subsubsection{Effect of Rayleigh number}

The horizon of predictability depends on Rayleigh number. For $R a=1 \times 10^{6}$ the horizon of predictability was 0.25 (Fig. 3a). For $R a=2 \times 10^{6}$ it decreased to 0.20 (Fig. 3b). For $R a=3 \times 10^{6}$ it decreased further to 0.17 (Fig. 3c), and for $R a=1 \times 10^{7}$ it is about 0.14 (Fig. 3d). As the Rayleigh 


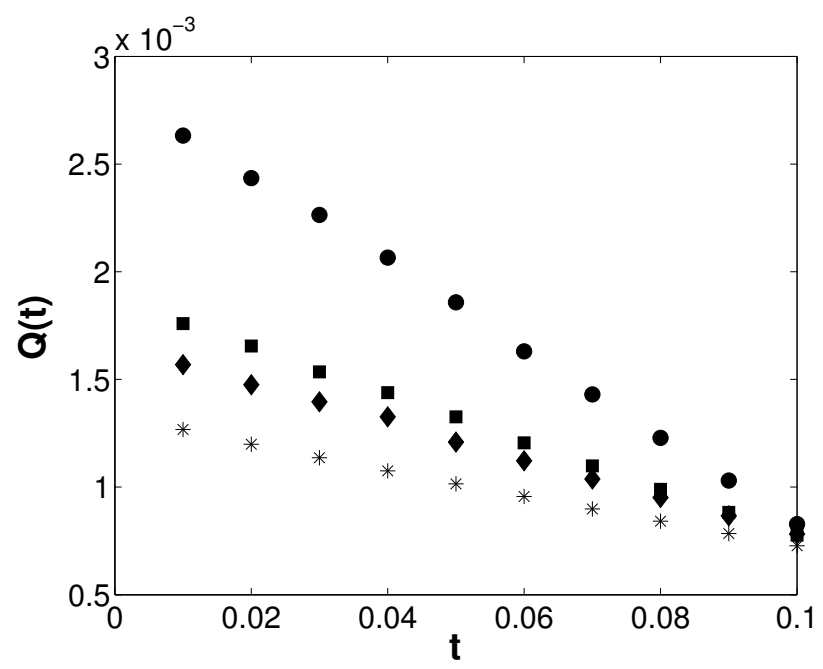

Fig. 5. Quality of the $4 \mathrm{D}-\mathrm{VAR}$ prediction, $Q(t)$, versus free-fall time, $t$. All plumes have a Prandtl number of 7 . Four different Rayleigh numbers are shown: $1 \times 10^{6}$, stars; $2 \times 10^{6}$, diamonds; $3 \times 10^{6}$, squares; $1 \times 10^{7}$, circles.

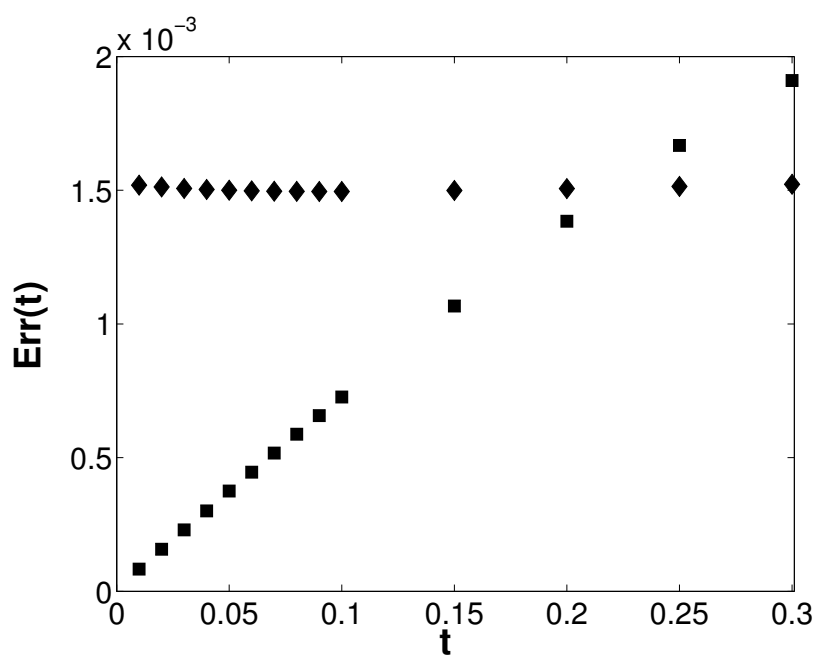

Fig. 6. Comparison of the DNS and 4D-VAR prediction for $R a=1 \times 10^{6}, \operatorname{Pr}=7$ with double resolution. DNS is shown with diamonds and $4 \mathrm{D}-\mathrm{VAR}$ with squares.

number increases, the nonlinear, inertial terms become more important, and the horizon of predictability decreases.

The quality of the 4D-VAR method is compared for different Rayleigh numbers in Fig. 5. We defined the quality of the $4 \mathrm{D}$-VAR prediction as:

$Q(t)=\operatorname{Err}(t)[\mathrm{DNS}]-\operatorname{Err}(t)[4 \mathrm{D}-\mathrm{VAR}]$.

The quality increases with the Rayleigh number for small forecast times. This is due to the fact that the inertia increases with the Rayleigh number. As the inertia increases, the plume becomes less sensitive to initial conditions. As the forecast time increases, the quality decreases. This decrease in quality, however, is less drastic for lower Rayleigh

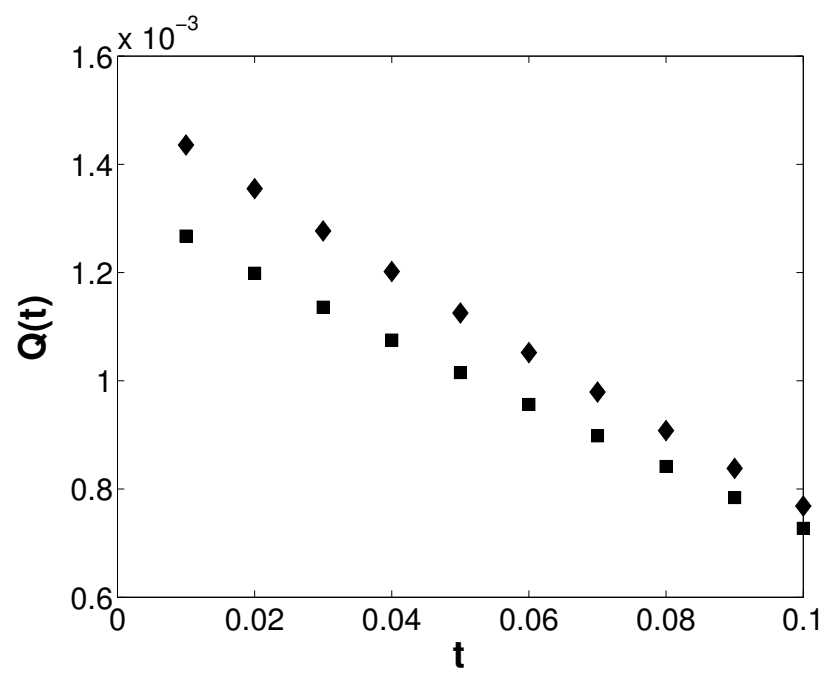

Fig. 7. Quality of the $4 \mathrm{D}-\mathrm{VAR}$ prediction, $Q(t)$ versus free-fall time, $t$. The Quality is defined as the difference between the error of the DNS and the 4D-VAR simulations. Data is shown $R a=1 \times 10^{6}$, $P r=7$. Single precision is shown by squares and double precision by diamonds.

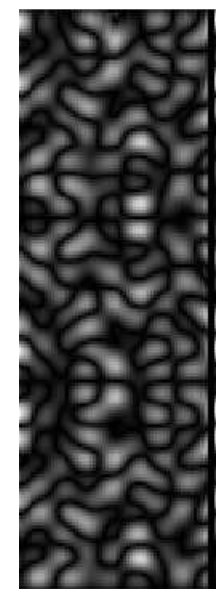

Fig. 8. A more random perturbation on the initial temperature conditions for $\operatorname{Ra}=1 \times 10^{6}, \operatorname{Pr}=7$. The initial conditions are the same as shown in Fig. 2a. Scale for the nondimensional temperature ranges from 0 for black to 0.06 for white.

numbers. This is due to the fact that the higher the inertia in a system, the more difficult it becomes to deterministically compute its exact state through time. Therefore, for the higher Rayleigh numbers the quality of the 4D-VAR prediction decreases more rapidly in time than for the lower Rayleigh numbers.

This means that a higher inertial system is not as likely to be affected by small fluctuations; therefore, it has better predictability (Lorenz, 1963). This is known as the butterfly effect. If a highly inertial system, such as the atmosphere, were very sensitive to small perturbations, a butterfly flapping its wings in Brazil could cause a tornado in Texas 


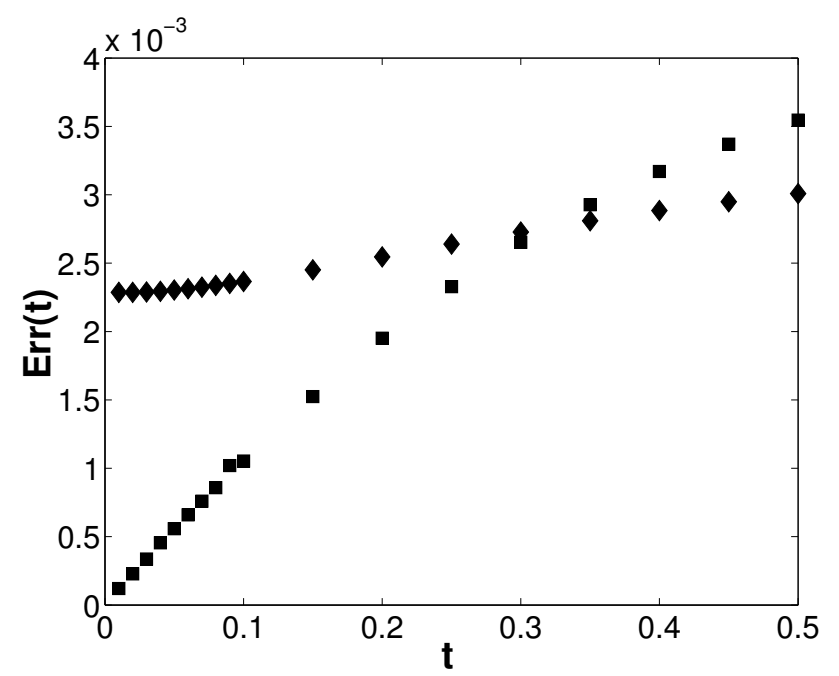

Fig. 9. Comparision of DNS and 4D-VAR prediction for a more random initial perturbation of a plume with $R a=1 \times 10^{6}, \operatorname{Pr}=7$. DNS prediction is shown by diamonds and $4 \mathrm{D}-\mathrm{VAR}$ prediction by squares.

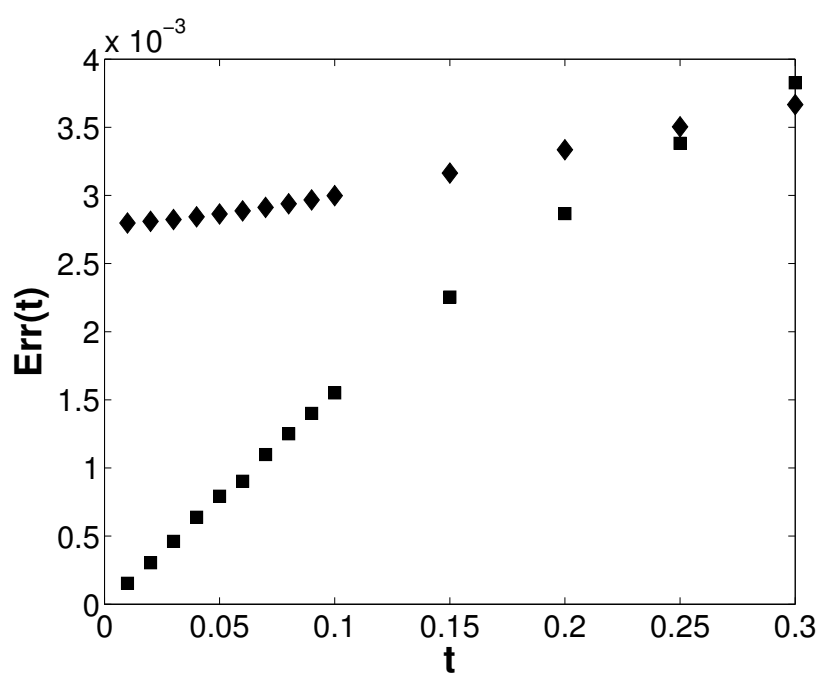

Fig. 10. Comparision of DNS and 4D-VAR prediction for a more random initial perturbation of a plume with $\operatorname{Ra}=2 \times 10^{6}, \operatorname{Pr}=7$. DNS prediction is shown by diamonds and $4 \mathrm{D}-\mathrm{VAR}$ prediction by squares.

(Lorenz, 1972). Although the quality of the prediction improves with $R a$, the horizon of predictability decreases with $R a$. This means that although we can generate better predictions for higher inertial systems, we cannot generate predictions for as long as we can for lower inertia systems.

\subsubsection{Effect of numerical resolution}

In order to test whether we used adequate numerical resolution in our simulations, we also ran the prediction for $R a=10^{6}, \operatorname{Pr}=7$ in double precision (Fig. 6). Our simulations were run using 32 bit single precision which gives 7 sig-

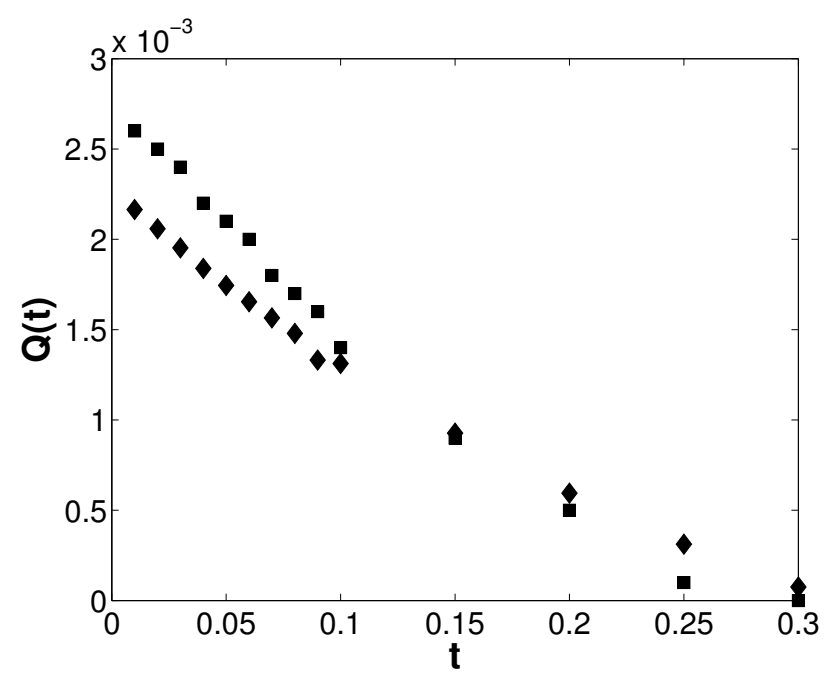

Fig. 11. Comparision of quality of 4D-VAR prediction for plumes of different Rayleigh number with a more random initial perturbation $R a=1 \times 10^{6}, P r=7$ is shown by diamonds, and $R a=2 \times 10^{6}$, $\operatorname{Pr}=7$ is shown by squares.

nificant digits on the IBM Regatta used in this study. There is a small decrease in the horizon of predictability from 0.25 to 0.22 indicating that it may be slightly more difficult to predict higher resolution simulations for a longer time period. We see that there is a $12 \%$ improvement in the quality of the prediction near $t \sim 0$ for the doubled numerical resolution (Fig. 7). The improvement in the quality of the prediction due to the increased numerical resolution, however, drops as the forecast time increases. This indicates that we can improve our short term predictions by using double precision, but that it does not tend to improve longer term predictions.

\subsubsection{Effect of initial perturbation}

We also tested the method with single precision using a more random perturbation of the initial conditions (Fig. 8). This perturbation was created by linear superposition of several different sinusoidal perturbations. We found that the horizon of predictability increased for $R a=1 \times 10^{6}, P r=7$ from 0.25 for the original perturbation to 0.30 for the more random perturbation (Fig. 9). For $R a=2 \times 10^{6}, \operatorname{Pr}=7$, the horizon of predictability increased from 0.20 to 0.25 (Fig. 10). There is also significant improvement in the quality of the predictions for the more random initial perturbation (Fig. 11) versus the more regular perturbation (Fig. 5). By perturbing different modes we do expect the response of the system to be slightly different due to the fact that each scale has an intrinsic horizon of predictability (Lorenz, 1969). However, the general conclusion still holds that the larger Rayleigh number flow has a shorter horizon of predictablity but a lower quality prediction. 


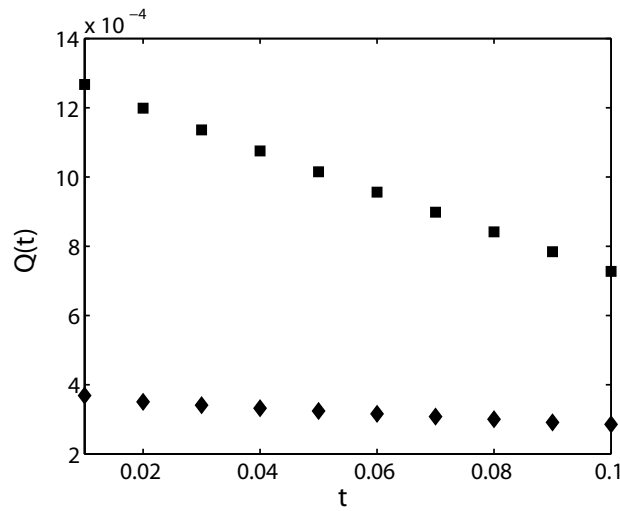

a)

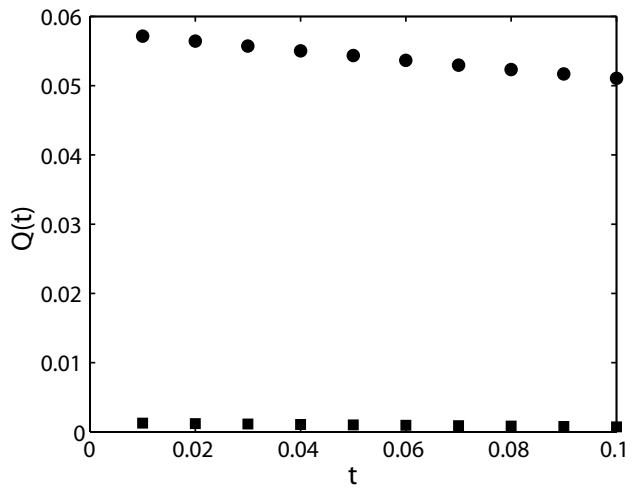

b)

Fig. 12. Quality of the $4 \mathrm{D}-\mathrm{VAR}$ prediction, $Q(t)$, versus free-fall time, $t$ for $R a=1 \times 10^{6}$. (a) Diamonds represent $P r=70$, and squares represent $\operatorname{Pr}=7$. (b) Diamonds represent $\operatorname{Pr}=7$, and squares represent $\operatorname{Pr}=0.7$.

\subsubsection{Effect of Prandtl number}

We next ran simulations at $R a=1 \times 10^{6}$ with $\operatorname{Pr}=0.7$ and 70 using single precision and the more regular perturbation. For a given $R a$, the 4D-VAR predictions are better for a lower $\mathrm{Pr}$ at small forecast times (Fig. 12). This is due to the fact that inertia strengthens as $\operatorname{Pr}$ decreases. We were only able to study plumes with Prandtl numbers up to 70 in this study. Previous studies of plume behavior with Prandtl numbers up to 20000 (Hier Majumder et al., 2004) have shown that there are still significant differences between finite and infinite Prandtl plumes even at these Rayleigh numbers. It would be interesting to conduct similar studies on the predictability of high and infinite Prandtl number plumes. Although our method can theoretically handle higher Prandtl numbers, it would be necessary to deal with the increasing stiffness of the finite Prandtl number convection equations along with the resulting large grid sizes. For example, plumes with Prandtl numbers on the order of $10^{4}$ at Rayleigh numbers of $10^{6}$ would require grid sizes of $512 \times 1536$ (Hier Majumder et al., 2004). Since each step of the forward solution is needed to solve the adjoint solution, the major computational expense of the adjoint method is the memory resources needed for storage of the forward solution (Daescu et al., 2003). Methods that have been developed for using the adjoint method with the stiff equations of atmospheric chemistry could prove useful for predictability studies of the large Prandtl numbers plumes (Elbern et al., 1997; Daescu et al., 2000; Sandu et al., 2003; Daescu et al., 2003).

\section{Conclusions}

We found that the 4D-VAR method is successful at correcting simulations that have erroneous initial conditions for finite Prandtl plumes. This technique can increase the ability to model plume phenomena for which observations, such as laboratory and seismic data, are available, but where the ex- act initial conditions are not well known. The 4D-VAR correction only works for a limited time, however. The time limit for the prediction is known as the predictability time.

We saw that as the inertia of the system increases with increasing Rayleigh number, the predictability time decreases. However, we also saw that we can generate better 4D-VAR predictions for higher inertial systems. The quality of the prediction increased with increasing $R a$ and decreasing $P r$. Therefore, we conclude that there is a trade-off in the finite Prandtl system. As inertia increases, the quality of the prediction increases, but the horizon of predictability decreases.

There are many other geophysical applications where a knowledge of the predictability is important. Water can significantly alter the behavior of mantle minerals. Water has a significant effect on the rheology of both olivine (Mei and Kohlstedt, 2000). Despite its importance in mantle dynamics, researchers are just beginning to develop models on how convection may distribute water in the mantle (Richard et al., 2002). We are currently working on applying this method to determine how well simulations can predict mantle water distribution despite a lack of knowledge about the initial distribution of water in the Earth's mantle.

The method in this study could also be adapted to sedimentary transport problems. In high Rayleigh number flow, such as in this study, diffusive processes are relatively weak. This is similar to the Navier-Stokes equation of turbulent flow in sediment transport where the viscous term is negligible in relation to the turbulent momentum exchange (Clifford and French, 1993).

Another potential use of the adjoint method would be in the modeling of sedimentary basins. Inverse methods have been used in the past to model the development of sedimentary basins. For example, reverse modeling has been used to restore the initial conditions of a sedimentary deposit before deformation due to Rayleigh-Taylor instabilities created by salt domes (Kaus and Podlachikov, 2001). Inverse methods and sensitivity analysis have also been used to 
help determine the deformation history of sedimentary basins (White and Bellingham, 2002; Bellingham and White, 2002). Since these types of studies are similar to the case of mantle convection discussed earlier (Bunge et al., 2003) in that the final state is observable, but the initial conditions are not well known, the use of the adjoint equations and variational data assimilation would be applicable to these types of studies.

Acknowledgements. We would like to thank B. Travis and S. Muhkerjee for many stimulating discussions on uses of the adjoint method. We would also like to thank J. Hernlund and an anonymous reviewer for their comments. A. Vincent acknowledges NSERC of Canada for providing support for this study.

Edited by: B. D. Malamud

Reviewed by: J. Hernlund and another referee

\section{References}

Bélanger, E. and Vincent, A.: Data assimilation (4D-VAR) to forecast flood in shallow waters with sediment erosion, J. Hydrol., 300, 114-125, 2005.

Bélanger, E., Vincent, A., and Fortin, A.: Data assimilation (4DVAR) for shallow-water flow: The case of the Chicoutimi River, Visual Geosciences, 8, 10.1007/s10 069-003-0009-7, 2003.

Bellingham, P. and White, N.: A two-dimensional inverse model for extensional sedimentary basins. 2. Application, J. Geophys. Res., 107, 10.1029/2001JB000 174, 2002.

Bunge, H.-P., Hagelberg, C. R., and Travis, B. J.: Mantle circulation models with variational data assimilation: Inferring past mantle flow and structure from plate motion histories and seismic tomography, Geophys. J. Int., 152, 280-301, 2003.

Burden, R. L. and Faires, J. D.: Numerical Analysis, PWS Publishing, Boston, 1993.

Cetegen, B. M., Dong, Y., and Soteriou, M. C.: Experiments on stability and oscillatory behavior of planar buoyant plumes, Phys. Fluids, 10, 1658-1665, 1998.

Clifford, N. J. and French, J. R.: Monitoring and modelling turbulent flow: Historical and contemporary perspectives, in: Turbulence: Perspectives on Flow and Sediment Transport, edited by: Clifford, N. J., French, J. R., and Hardisty, J., pp. 1-34, John Wiley \& Sons, New York, 1993.

Courtier, P.: Variational Methods, in: Data Assimilation in Oceanography: Theory and Practice, edited by: Ghil, M., Ide, K., Bennett, A., Courtier, P., Kimoto, M., Nagata, M., Saiki, M., and Sato, N., pp. 211-218, Meteorological Society of Japan, Tokyo, 1997.

Courtier, P., Derber, J., Errico, R., Louis, J.-F., and Vukićević, T.: Important literature on the use of adjoint methods, variational methods and the Kalman filter in meteorology, Tellus, 45A, 342357, 1993.

Daescu, D., Carmichael, G. R., and Sandu, A.: Adjoint implementation of Rosenbrock methods applied to variational data assimilation problems, J. Comput. Phys., 165, 496-510, 2000.

Daescu, D. N. and Navon, I. M.: Adaptive observations in the context of the 4D-Var data assimilation method, Meteorol. Atmos. Phys., 85, 205-226, 2003.

Daescu, D. N., Sandu, A., and Carmichael, G. R.: Direct and adjoint sensitivity analysis of chemical kinetic systems with KPP: Part II-numerical validation and applications, Atmos. Environ., 37, 5097-5114, 2003.
Daley, R.: Atmospheric Data Analysis, Cambridge University Press, Cambridge, 1991.

Dimet, F.-X. L. and Talagrand, O.: Variational algorithms for analysis and assimilation of meteorological observations: Theoretical aspects, Tellus, 38A, 97-110, 1986.

Ehrendorfer, M.: Four-dimensional data assimilation: Comparison of variational and sequential algorithms, Quarterly Journal of the Royal Meteorological Society, 118, 673-713, 1992.

Elbern, H., Schmidt, H., and Ebel, A.: Variational data assimilation for tropospheric chemistry and modeling, J. Geophys. Res., 102, 15 967-15 985, 1997.

Errico, R. M.: What is an adjoint model?, Bulletin of the American Meteorological Society, 78, 2577-2591, 1997.

Hier Majumder, C. A., Yuen, D. A., and Vincent, A. P.: Four dynamical regimes for a starting plume model, Phys. Fluids, 16, 1516-1531, 2004.

Ismail-Zadeh, A., Schubert, G., Tsepelev, I., and Korotkii, A.: Inverse problem of thermal convection: numerical approach and application to mantle plume restoration, Physics of the Earth and Planetary Interiors, 145, 99-114, 2004.

Kaminski, E. and Jaupart, C.: Laminar starting plumes in highPrandtl-number fluids, J. Fluid Mechanics, 478, 287-298, 2003.

Kaus, B. J. and Podlachikov, Y. Y.: Forward and Reverse modeling of the three-dimensional viscous Rayleigh-Taylor instability, Geophys. Res. Lett., 28, 1095-1098, 2001.

Lavelle, J. W.: Buoyancy-driven plumes in rotating, stratified cross flows: Plume dependence on rotation, turbulent mixing, and cross-flow strength, J. Geophys. Res., 102, 3405-3420, 1997.

Lions, J. L.: Contrôle Optimal de Systèmes Gouvernés par des Équations aux Dérivées Partielles, Dunod, Paris, 1968.

Lithgow-Bertelloni, C., Richards, M. A., Conrad, C. P., and Griffiths, R. W.: Plume generation in natural thermal convection at high Rayleigh and Prandtl numbers, J. Fluid Mechanics, 43, 121, 2001.

Lorenz, E. N.: Deterministic nonperiodic flow, Journal of the Atmospheric Sciences, 20, 130-141, 1963.

Lorenz, E. N.: The predictability of a flow which possesses many scales of motion, Tellus, 21, 289-307, 1969.

Lorenz, E. N.: Predictability: Does the flap of a butterfly's wings in Brazil set off a tornado in Texas?, American Association for the Advancement of Science Annual Meeting Programs, 139, 1972.

Lorenz, E. N.: Atmoshperic predictability experiments with a large numerical model, Tellus, 34, 505-513, 1982.

Lorenz, E. N.: Estimates of atmospheric predictability at medium range, in: Predictability of Fluid Motions, edited by: Holloway, G. and West, B. J., pp. 133-139, American Institute of Physics, New York, Conference Proceedings Number 106, 1984.

Marchuk, G. I.: Adjoint Equations and Analysis of Complex Systems, Kluwer Academic Publishers, Dordrecht, 1995.

Mei, S. and Kohlstedt, D. L.: Influence of water on plastic deformation of olivine-basalt aggregates, J. Geophys. Res., 105, 21471 $21481,2000$.

Montelli, R., Nolet, G., Dahlen, F. A., Masters, G., Engdahl, R. E., and Hung, S.-H.: Finite-frequency tomography reveals a variety of plumes in the mantle, Science, 303, 338-343, 2004.

Moses, E., Zocchi, G., and Libchaber, A.: An experimental study of laminar plumes, J. Fluid Mechanics, 251, 581-601, 1993.

Neupauer, R. M. and Wilson, J. L.: Adjoint-derived location and travel time probabilities for a multidimensional groundwater system, Water Resour. Res., 37, 1657-1668, 2001.

Rast, M. P.: Compressible plume dynamics and stability, J. Fluid Mechanics, 369, 125-149, 1998. 
Rast, M. P.: Solar granulation: A surface phenomenon, in Geophysical and Astrophysical Convection, edited by P. A. Fox and R. M. Kerr, pp. 199-219, Gordon Breach Scientific Publisher, New York, 2000

Richard, G., Monnereau, M., and Ingrin, J.: Is the transition zone an empty water reservoir? Inferences from numerical model of mantle dynamics, Earth Plan. Sci. Lett., 205, 37-51, 2002.

Roache, P. J.: Computational Fluid Dynamics, Hermosa, Albuquerque, 2nd edn., 1976.

Sanders, B. F. and Katopodes, N. D.: Control of canal flow by adjoint sensitivity method, Journal of Irrigation and Drainage Engineering, 125, 287-297, 1999.

Sanders, B. F. and Katopodes, N. D.: Adjoint sensitivity analysis for shallow-water wave control, Journal of Engineering Mechanics, 126, 909-919, 2000.

Sandu, A., Daescu, D. N., and Carmichael, G. R.: Direct and adjoint sensitivity analysis of chemical kinetic systems with KPP: Part I-theory and software tools, Atmos. Environ., 37, 5083-5096, 2003.
Schröter, J., Seiler, U., and Wenzel, M.: Variational assimilation of GEOSAT data into an eddy-resolving model of the Gulf Stream extension area, Journal of Physical Oceanography, 23, 925-953, 1993.

Talagrand, O. and Courtier, P.: Variational assimilation of meteorological observations with the adjoint vorticity equation. I: Theory, Quarterly Journal of the Royal Meteorological Society, 113, 1311-1328, 1987.

Tritton, D. J.: Physical Fluid Dynamics, Oxford University Press, Oxford, 2nd edn., 1988.

White, N. and Bellingham, P.: A two-dimensional inverse model for extensional sedimentary basins. 1. Theory, J. Geophys. Res., 107, 10.1029/2001JB000 173, 2002.

Zhao, D.: Seismic structure and origin of hotspots and mantle plumes, Earth Plan. Sci. Lett., 192, 251-265, 2001.

Zhu, Y., Todling, R., Guo, J., Cohen, S. E., Navon, I. M., and Yang, Y.: The GEOS-3 retrospective data assimilation system: The 6hour lag case, The Monthly Weather Review, 131, 2129-2150, 2003. 